\title{
Nanoporous Membranes Enable Concentration and Transport in Fully Wet Paper-Based Assays
}

\author{
Max M. Gong, ${ }^{\dagger}$ Pei Zhang, ${ }^{\dagger}$ Brendan D. MacDonald, ${ }^{*},{ }^{\dagger}$ and David Sinton*, ${ }^{\dagger}$ \\ ${ }^{\dagger}$ Department of Mechanical and Industrial Engineering, University of Toronto, 5 King's College Road, Toronto, Ontario M5S 3G8, \\ Canada \\ ${ }^{\ddagger}$ Faculty of Engineering and Applied Science, University of Ontario Institute of Technology, 2000 Simcoe Street North, Oshawa, \\ Ontario L1H 7K4, Canada
}

\section{Supporting Information}

\begin{abstract}
Low-cost paper-based assays are emerging as the platform for diagnostics worldwide. Paper does not, however, readily enable advanced functionality required for complex diagnostics, such as analyte concentration and controlled analyte transport. That is, after the initial wetting, no further analyte manipulation is possible. Here, we demonstrate active concentration and transport of analytes in fully wet paper-

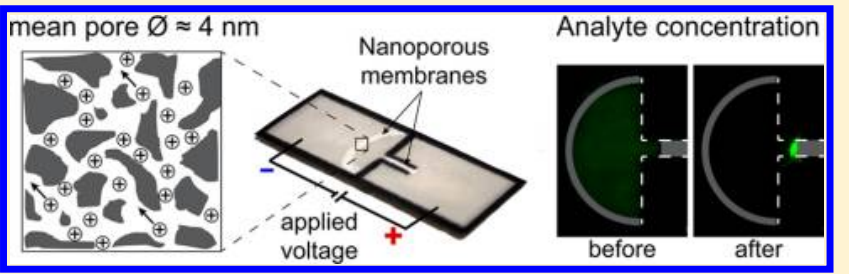
based assays by leveraging nanoporous material (mean pore diameter $\approx 4 \mathrm{~nm}$ ) and ion concentration polarization. Two classes of devices are developed, an external stamp-like device with the nanoporous material separate from the paper-based assay, and an in-paper device patterned with the nanoporous material. Experimental results demonstrate up to 40-fold concentration of a fluorescent tracer in fully wet paper, and directional transport of the tracer over centimeters with efficiencies up to $96 \%$. In-paper devices are applied to concentrate protein and colored dye, extending their limits of detection from $\sim 10$ to $\sim 2 \mathrm{pmol} / \mathrm{mL}$ and from $\sim 40$ to $\sim 10 \mu \mathrm{M}$, respectively. This approach is demonstrated in nitrocellulose membrane as well as paper, and the added cost of the nanoporous material is very low at $\sim 0.015$ USD per device. The result is a major advance in analyte concentration and manipulation for the growing field of low-cost paperbased assays.
\end{abstract}

$\mathrm{P}$ aper has been used as a platform for many micro- and nanoscale applications including paper-based electronics, ${ }^{1-5}$ plasmonics, ${ }^{6-9}$ and microfluidics. ${ }^{10-14}$ Several factors motivate the recent surge in paper applications: (1) abundance and low cost, (2) compatibility with biological samples, (3) ease of fabrication and modification, and (4) ease of disposability. ${ }^{10-12}$ In the context of paper-based microfluidic assays, the lateral flow assay is a well-established and historic format for routine point-of-care diagnostics. ${ }^{12}$ Commercial lateral flow assays are available for testing a wide spectrum of biomarkers, requiring minimal expertise and equipment. Perhaps the most widely used lateral flow assay is the home pregnancy test, enabling end users to self-test for pregnancy at their own discretion. Fluid transport (and associated analyte transport) in paper-based microfluidic assays occurs via passive capillary action and depends on the physical properties of paper (i.e., surface area, thickness, porosity, and pore size). ${ }^{12,15}$ Flow control in paper-based assays is critical for enhancing detection sensitivity via analyte concentration, and for coordinating and timing the delivery of samples or reagents.

Existing strategies for analyte concentration during paper wetting consist of chemical amplification using gold nanoparticles, ${ }^{16-18}$ molecular amplification using nucleic acid circuits, ${ }^{19}$ and lateral flow concentration leveraging the evaporation of a volatile solvent. ${ }^{20}$ Strategies for controlled analyte transport during wetting include: varying the geometry and dimensions of paper reservoirs and channels; ${ }^{21,22}$ actuating paper cantilevers to connect channels; ${ }^{23,24}$ sliding a paper test strip through a stationary reagent loading unit; ${ }^{25}$ and adding soluble physical barriers to act as time delays ${ }^{26-28}$ or as shut-off valves. ${ }^{29,30}$ These approaches have demonstrated enhanced flow control; however, they rely on capillary forces generated in remaining dry portions of the paper matrix.

Once a paper-based assay is saturated, further analyte concentration or transport is generally not possible. To achieve postwetting manipulation, the most relevant available techniques are electrokinetic in nature. Electrophoretic separations have been demonstrated in fully wet paper. ${ }^{31}$ Droplet movement has also been demonstrated with electrowetting on a dielectric paper surface. ${ }^{32}$ Concentration and directional transport of analytes using electrokinetic phenomena have yet to be demonstrated. Ion concentration polarization (ICP) is another promising approach for analyte manipulation within the electrokinetic toolbox. ICP occurs at the interface of microfluidic and ion selective nanofluidic channels under an applied electric field. ${ }^{33}$ An ion depletion region forms at the interface, which repels charged particles. ${ }^{34}$ ICP has been used extensively for analyte concentration ${ }^{35-40}$ and separation, ${ }^{41}$ and

Received: March 17, 2014

Accepted: July 21, 2014

Published: July 21, 2014 
water desalination ${ }^{42,43}$ in conventional microfluidic channels. ICP-induced $\mathrm{pH}$ changes at the micronano interface have also been studied in detail. ${ }^{44}$ This phenomenon has yet to be leveraged in alternative microfluidic formats, such as paper, for analyte concentration and controlled analyte transport. The potential for patterning nanoporous membranes within microporous paper presents an opportunity to build ICP functionality into paper-based assays.

Here, we demonstrate active concentration and transport of analytes in fully wet paper-based assays by leveraging nanoporous material (mean pore diameter $\approx 4 \mathrm{~nm})^{45}$ and ion concentration polarization. Two classes of devices are developed, an external stamp-like device with the nanoporous material separate from the paper-based assay, and an in-paper device patterned with the nanoporous material. Experimental results demonstrate up to 40 -fold concentration of a fluorescent tracer in fully wet paper, and directional transport of the tracer over centimeters with efficiencies up to $96 \%$. In a diagnostic application, in-paper devices are used to concentrate fluorescein isothiocyanate conjugate bovine serum albumin (FITCalbumin) and bromocresol green dye, extending their limits of detection from $\sim 10$ to $\sim 2 \mathrm{pmol} / \mathrm{mL}$ and from $\sim 40$ to $\sim 10$ $\mu \mathrm{M}$, respectively.

\section{EXPERIMENTAL SECTION}

Device Classification. Two classes of devices are developed, an external stamp-like device with the nanoporous membranes separate from the paper-based assay, and an inpaper device. Nanoporous membranes are embedded into a silicone rubber layer in the external devices to serve as the membrane-paper interface for inducing ICP. Nanoporous membranes are directly patterned into the in-paper devices, serving two important functions: (1) they act as hydrophobic barriers, much like wax, to define fluidic reservoirs and channels, and (2) they form the micronano interface necessary for ICP. The utility of the nanoporous membranes patterned into paper is similar to that of the external devices, where the presence of paper fibers does not compromise membrane function (i.e., cation selective transport through the nanoporous membranes is maintained in both cases).

Fabrication and Operation of the External Devices. Schematics for the external concentration and transport devices are shown in Figure $1 \mathrm{a}$ and $\mathrm{b}$, with the central ICP phenomenon depicted in Figure 1c. The fabrication and assembly procedures for the external devices are detailed below and also in Figure S1 of the Supporting Information. The geometry for the external devices was first drawn in AutoCAD and then cut into the relevant layers using a $\mathrm{CO}_{2}$ laser printer (Universal Laser Systems Inc., AZ). The Nafion nanoporous membrane layer was fabricated by cutting through $0.5 \mathrm{~mm}$ thick silicone rubber (McMaster Carr, $\mathrm{OH}$ ) with laser writing power of $50 \%$ and speed of $2.0 \%$, producing $\sim 0.3 \mathrm{~mm}$ wide throughcuts after 15 passes. Through-cuts for buffer reservoirs were micromachined into $3.0 \mathrm{~mm}$ thick poly(methyl methacrylate) (PMMA) (Plastic World, Toronto, Canada) with laser writing power of $100 \%$ and speed of $1.0 \%$ after one pass, having an enlarged geometry of the through-cuts in the silicone layer (Figure 1a and b). Through-holes for bolts were also micromachined into the silicone and PMMA layers for device assembly. After these cutting steps, the prepared silicone was elevated onto rulers, and a pipet was used to fill the throughcuts with $20 \mu \mathrm{L}$ of Nafion 117 solution, $5 \%$ in a mixture of lower aliphatic alcohols (Sigma-Aldrich, MO), followed by

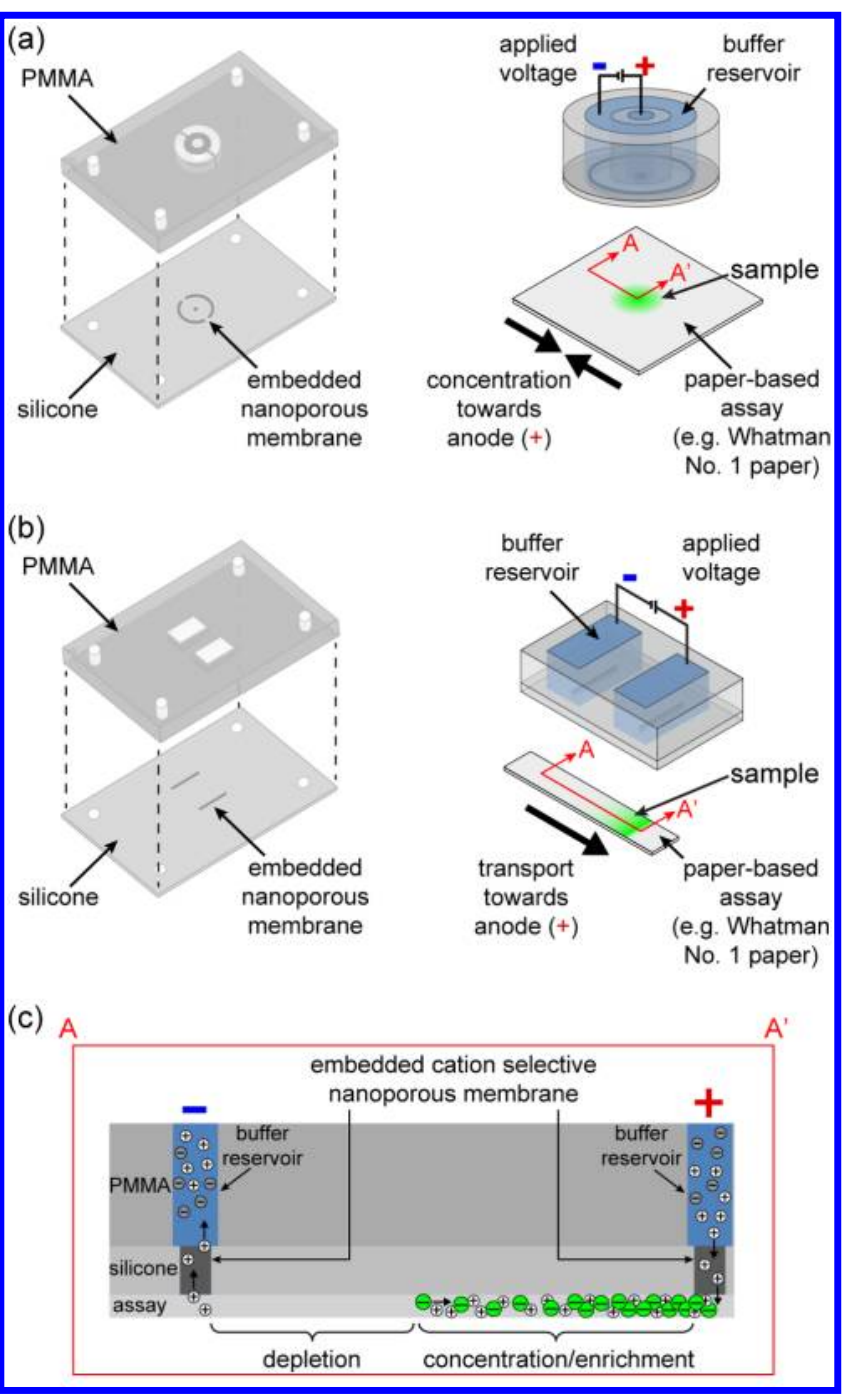

Figure 1. External devices for analyte concentration and transport. Schematics of the external (a) concentration and (b) transport devices. Nafion nanoporous membranes are embedded into the silicone layer to trigger ICP at the membrane-paper interface. (c) Cross-section of the devices and paper-based assays depicting ICP under an applied voltage. Ion depletion occurs at the anodic side of the nanoporous membrane to push analytes toward the anode.

heating in an oven for $30 \mathrm{~min}$ at $65{ }^{\circ} \mathrm{C}$. The silicone layer was then placed in a Petri dish and immersed in $0.25 \times$ Tris-acetateEDTA buffer (TAE buffer, $10 \mathrm{mM}$ Tris-acetate, and $0.25 \mathrm{mM}$ EDTA at $\mathrm{pH} 8.2$ ) for $30 \mathrm{~min}$ to hydrate the hardened Nafion. The silicone and PMMA layers were assembled together using bolts and nuts to form a complete device.

To operate, the assembled external device is simply brought into contact with the paper-based assay, buffer reservoirs are filled with $0.25 \times$ TAE buffer, and the voltage applied, as shown in Figure 1 and Supporting Information Figure S1. Importantly, the silicone layer containing the embedded nanoporous membranes prevents direct mixing of the sample in the assay with buffer in the buffer reservoirs. Under an applied voltage, ions migrate within the assay to their respective counterelectrodes. Figure 1c depicts this phenomenon in the crosssection of the whole device. Cations in the assay migrate toward the negative electrode (cathode) and travel through the cation selective Nafion nanoporous membrane into a buffer reservoir, while anions (e.g., fluorescent tracer and all other negatively 
charged species in solution) migrate toward the positive electrode (anode) through the assay, stopping at the membrane-paper interface. The efflux of cations from the anodic side of the nanoporous membrane causes anions to also vacate the vicinity to conform to electrical neutrality, forming an ion depletion region. This ion depletion region pushes and concentrates anions toward the anode. Conversely, enrichment of anions occurs at the cathodic side of the nanoporous membrane due to the influx of cations. In the external concentration device, enrichment is leveraged at the inner membrane and ion depletion at the outer membrane. The geometry of the membranes is symmetric for the transport device, where direction is controlled by adjusting the polarity of the membranes. We envision the external devices to be used as portable sample preparation tools in the field or clinic where conventional laboratory equipment may not be readily available. For example, the external concentration device can be used to preconcentrate sample in an assay in lieu of centrifugation and sample extraction. These devices can be used multiple times when properly sterilized after each use (i.e., contact surface is rinsed with deionized water, wiped dry, and then wiped with ethanol).

Fabrication and Operation of the In-Paper Devices. Schematics for the in-paper concentration and transport devices are shown in Figure $2 \mathrm{a}$ and $\mathrm{b}$, with the ICP phenomenon depicted in Figure 2c. The in-paper devices are the twodimensional analogues of the external devices, where nanoporous membranes are directly patterned into the paper. The patterned nanoporous membranes serve (1) to act as

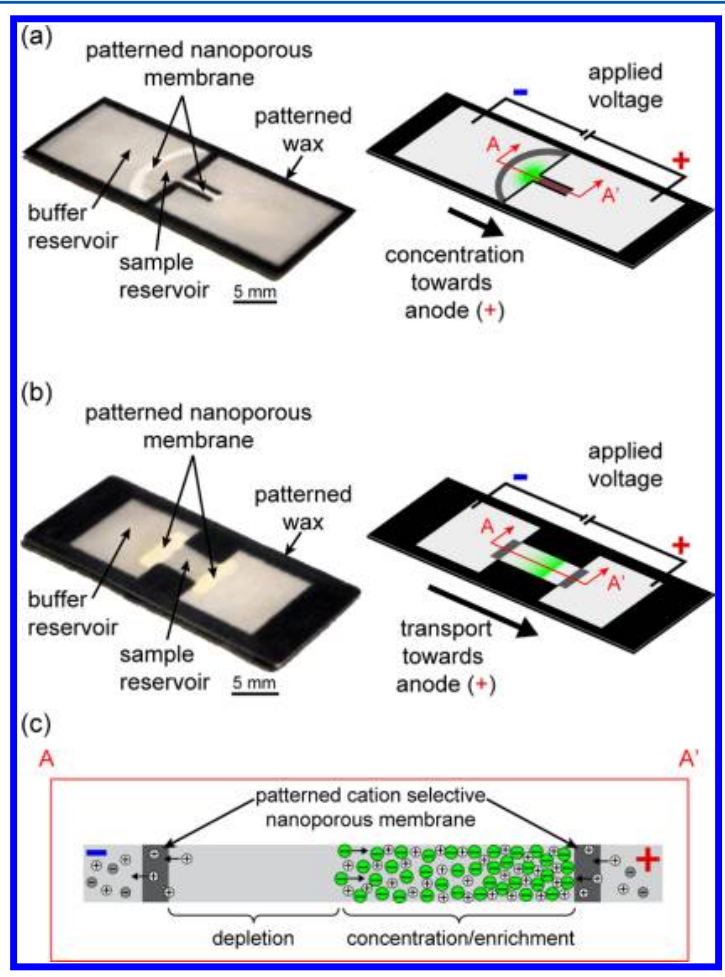

Figure 2. In-paper devices for analyte concentration and transport. Schematics of the in-paper (a) concentration and (b) transport configurations. Fluidic regions are defined by Nafion nanoporous membranes and wax patterned into the paper. (c) Cross-section of the in-paper devices illustrating ICP under an applied voltage. Ion depletion occurs at the anodic side of the nanoporous membrane to push analytes toward the anode. hydrophobic barriers, much like wax, to define fluidic reservoirs and channels, and (2) to create the micronano interfaces necessary for ICP. The in-paper devices were fabricated from $0.18 \mathrm{~mm}$ thick Whatman No. 1 chromatography paper (SigmaAldrich, MO). A Xerox ColorQube 8570N color printer (Xerox Canada, ON, Canada) was used to pattern the wax boundary, and the patterned paper was heated at $150{ }^{\circ} \mathrm{C}$ for $2 \mathrm{~min}$ to allow penetration of the wax through the thickness of the paper. Nanoporous membranes were patterned into the paper by manually depositing Nafion 117 solution via a pipet, $\sim 3 \mu \mathrm{L}$ of Nafion per device. Devices were then soaked in $0.25 \times \mathrm{TAE}$ buffer for $30 \mathrm{~min}$ to hydrate the membrane. Following membrane hydration, devices were air-dried at room temperature, and rehydrated on use. The morphology of hydrated Nafion has been well-characterized in the literature, ${ }^{45}$ where the mean pore diameter is $\sim 4 \mathrm{~nm}$, well-suited to inducing ICP phenomena. Importantly, the sulfonic acid groups in Nafion provide a net negative surface charge in the pores, ${ }^{45}$ enabling the selective transport of cations through the membrane during ICP. The analytes used here, as specified below, are negatively charged at their respective $\mathrm{pH}$ values and do not pass through the cation selective membranes patterned into the in-paper devices, but rather migrate within the paper matrix and concentrate at the membrane-paper interface when ICP is induced, as shown in Figure 2c.

Operation of these devices is the same as the external ones: a voltage is applied across the device at the buffer reservoirs (as depicted in Figure $2 a$ and $b$ ) to trigger ion depletion in the sample region bounded by the nanoporous membranes (as depicted in Figure 2c). The in-paper approach offers advanced functionality in a disposable unit at low cost and potential for scale-up. The added cost of Nafion in paper is very low at $\sim 0.015$ USD per device, given a material cost of $\sim 0.005$ USD/ $\mu \mathrm{L}$ (Sigma-Aldrich, MO). The total material cost per device, using No. 1 chromatography paper, is $\sim 0.03$ USD (a detailed breakdown of material costs and fabrication time requirements is provided in Table S1 and Table S2 of the Supporting Information). We envision an extension in functionality of currently available lateral flow assays and paper-based colorimetric assays by integrating nanoporous membranes into these assays using the approach described above. Specifically, substantial improvement in the sensitivity of existing assays can be potentially achieved using our concentration method.

Preparation of Fluorescein, Protein, and Dye Solutions. Fluorescein solution was prepared by mixing fluorescein in $0.25 \times$ TAE buffer $(10 \mathrm{mM}$ Tris-acetate and $0.25 \mathrm{mM}$ EDTA at $\mathrm{pH} 8.2$ ). The solution was adjusted to a working $\mathrm{pH}$ of 9.0 using $1 \times \mathrm{NaOH}$ solution. This adjustment was done to minimize loss in the emission intensity of fluorescein during experimentation, where intensity can drop for $\mathrm{pH}<8 .^{46}$ Tests were conducted at both $\mathrm{pH} 8.2$ and 9.0 with no observable differences in the buffer capacity, as indicated by consistent fluorescein migration in both cases. Fluorescein isothiocyanate conjugate bovine serum albumin (FITCalbumin) was diluted with $0.25 \times$ TAE buffer into molar concentrations ranging from 2 to $30 \mathrm{pmol} / \mathrm{mL}$ (i.e., 2, 4, 8, 11, 15 , and $30 \mathrm{pmol} / \mathrm{mL}$ ). Bromocresol green dye was diluted with $0.25 \times \mathrm{TAE}$ buffer into molar concentrations ranging from 10 to $100 \mu \mathrm{M}$ (i.e., 10, 25, 50, and $100 \mu \mathrm{M}$ ). All reagents were obtained from Sigma-Aldrich.

Data Acquisition and Analysis. An inverted fluorescence microscope (DMI 6000B, Leica Microsystems Inc., ON, 
Canada) was used to capture images of fluorescein concentration and transport, and FITC-albumin concentration. Image sequences were taken with a CCD camera (Orca AG, Hamamatsu, NJ). Captured fluorescence images were background subtracted using a dark field image. A desktop scanner (CanoScan 9000F, Canon Canada Inc., ON, Canada) was used to image bromocresol green dye concentration. All captured images were processed in ImageJ and quantified using Excel and Mathematica.

\section{RESULTS AND DISCUSSION}

Active Concentration and Transport Using the External Device. Experimental results for fluorescent tracer concentration using the external concentration device are shown in Figure 3. For the circular device geometry (overlaid in Figure 3a), an ion depletion region pushes the tracer radially inward from the outer membrane, while enrichment occurs at the center, underneath the inner membrane. The diameters of the inner and outer membranes are fabricated as 0.12 and 8 $\mathrm{mm}$, respectively. For an applied voltage of $50 \mathrm{~V}$, a $15 \mu \mathrm{L}$ sample of fluorescein solution is concentrated in a $10 \mathrm{~mm} \times 10$ $\mathrm{mm}$ paper sheet after $3 \mathrm{~min}$ (movie 1 in the Supporting Information). In Figure 3a, the diameter of the focused area is $1.2 \mathrm{~mm}$ from an initial diameter of $8 \mathrm{~mm}$, which equates to a surface area ratio of 44 -fold. Specifically, 44 -fold provides an upper limit on the concentration factor possible with this geometry. The maximum peak concentration factor, in Figure $3 \mathrm{~b}$, is 40 -fold ( $91 \%$ of the upper limit), as quantified by the ratio of final to initial peak fluorescence intensity.

Figure 3a shows several notable time points: (1) fluorescein enrichment at the center is first observed at $t=15 \mathrm{~s}$, (2) concentration due to ion depletion becomes visible at $t=50 \mathrm{~s}$ (i.e., propagating wave from the outer membrane), (3) ion depletion and enrichment regions merge at $t=105 \mathrm{~s}$ (also specified in Figure $3 \mathrm{~b}$ by the left dashed line), and (4) concentration reaches saturation at $t=155 \mathrm{~s}$ (also specified in Figure $3 b$ by the right dashed line). For the intensity profiles in Figure $3 \mathrm{c}$, outer peaks at $t=105 \mathrm{~s}$ denote the merging of the ion depletion and enrichment regions. These peaks become pronounced at the end of the concentration process at $t=155$ s, resulting in a concentrated ring. Collectively, the results in Figure 3 demonstrate a substantial improvement in signal intensity after analyte concentration in fully wet paper.

Experimental results for fluorescent tracer transport using the external transport device are shown in Figure 4. For an applied voltage of $50 \mathrm{~V}$, a $5 \mu \mathrm{L}$ sample of fluorescein solution is transported multiple cycles in a $6 \mathrm{~mm} \times 3 \mathrm{~mm}$ paper strip (movie 2 in the Supporting Information). The sample is initially concentrated at an average rate of $1.6 \mathrm{~mm} / \mathrm{min}$ from the cathode to the anode, as shown in Figure 4a. Subsequent transport cycles in predetermined directions are achieved by simply switching the polarity of the membranes (i.e., switching the onset location of ion depletion), as shown in Figure $4 \mathrm{~b}$ and c. Notably, the repeated transport is achieved over the $\sim 6 \mathrm{~mm}$ distance (total of $\sim 18 \mathrm{~mm}$ ) with minimal loss. The full width at half-maximum (fwhm) values for the initial and two subsequent transport cycles are $0.38,0.34$, and $0.26 \mathrm{~mm}$. Thus, from an initially focused sample, the peak is broadened as it translates through the paper and then refocused with negligible broadening and little net loss.

Transport efficiency is quantified by comparing the area under the curve (AUC) of the final state in each cycle to the AUC of the final state in the initial concentration step (i.e.,
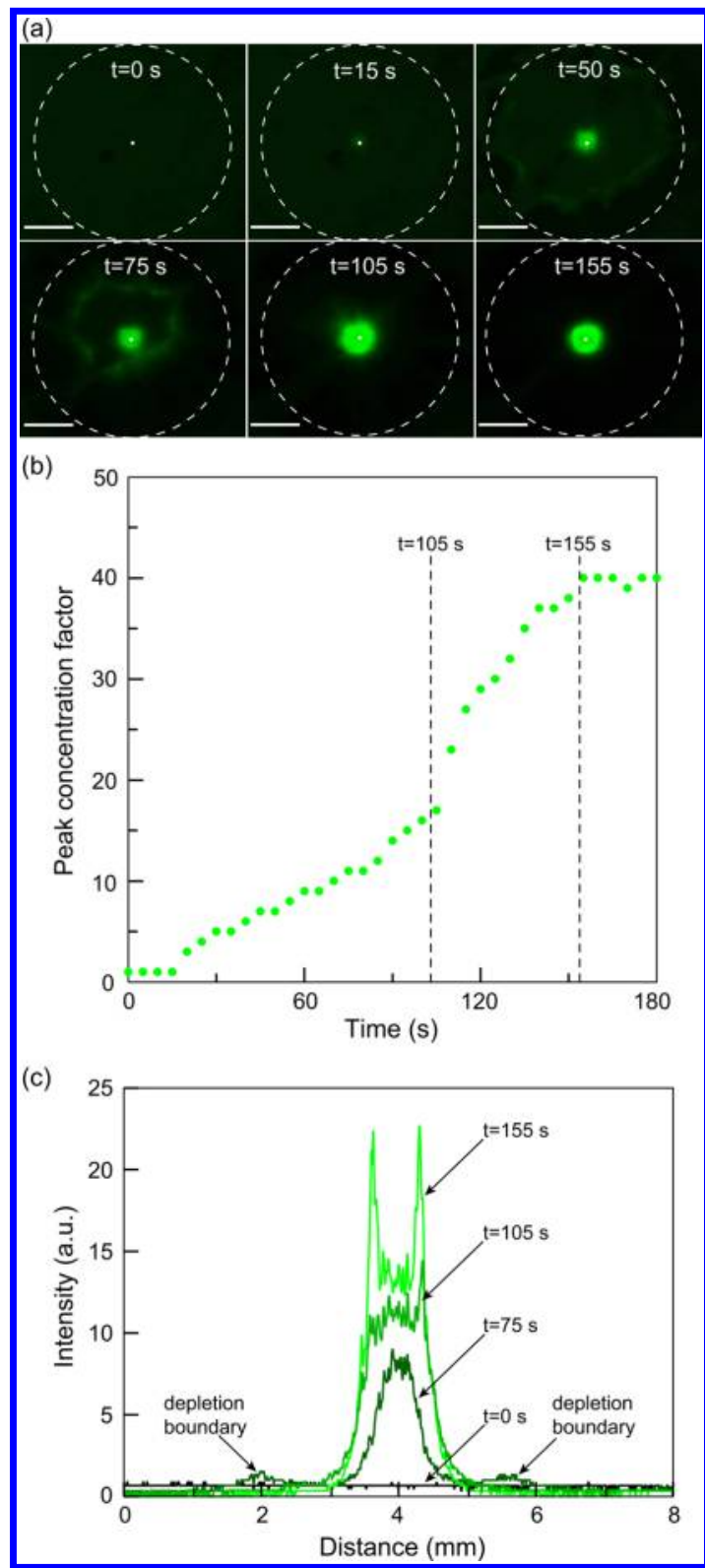

Figure 3. Active concentration using the external device. (a) Contrast enhanced images of fluorescent tracer concentration under an applied voltage of $50 \mathrm{~V}$. The location of the outer membrane is indicated by the dashed circle and the inner membrane by the dot. (b) Time evolution of peak concentration factor (i.e., ratio of final to initial peak fluorescence intensity). The maximum value reaches 40 -fold at $t=155$ s. Merging of the ion depletion and enrichment regions is indicated by the left dashed line at $t=105 \mathrm{~s}$. The peak concentration factor starts to plateau at $t=155 \mathrm{~s}$, as indicated by the right dashed line. (c) Intensity profiles at selected time points, as indicated. Scale bars are $2 \mathrm{~mm}$.

comparison of AUC in state IV in Figure $4 \mathrm{~b}$ and $\mathrm{c}$ to the AUC in state IV in Figure 4a). A transport efficiency of $\sim 96 \%$ is observed for the first cycle, decreasing to $\sim 88 \%$ after the second cycle. The average transport rate also decreases from 0.8 to $0.6 \mathrm{~mm} / \mathrm{min}$ for the first and second cycles, respectively. These small reductions in transport efficiency and rate can be attributed to lowered ionic current (i.e., ion migration) as a result of fluid evaporation from the paper, as well as some inherent retention of tracer in the paper. Overall, the results in Figure 4 demonstrate the effective transport of analytes over 


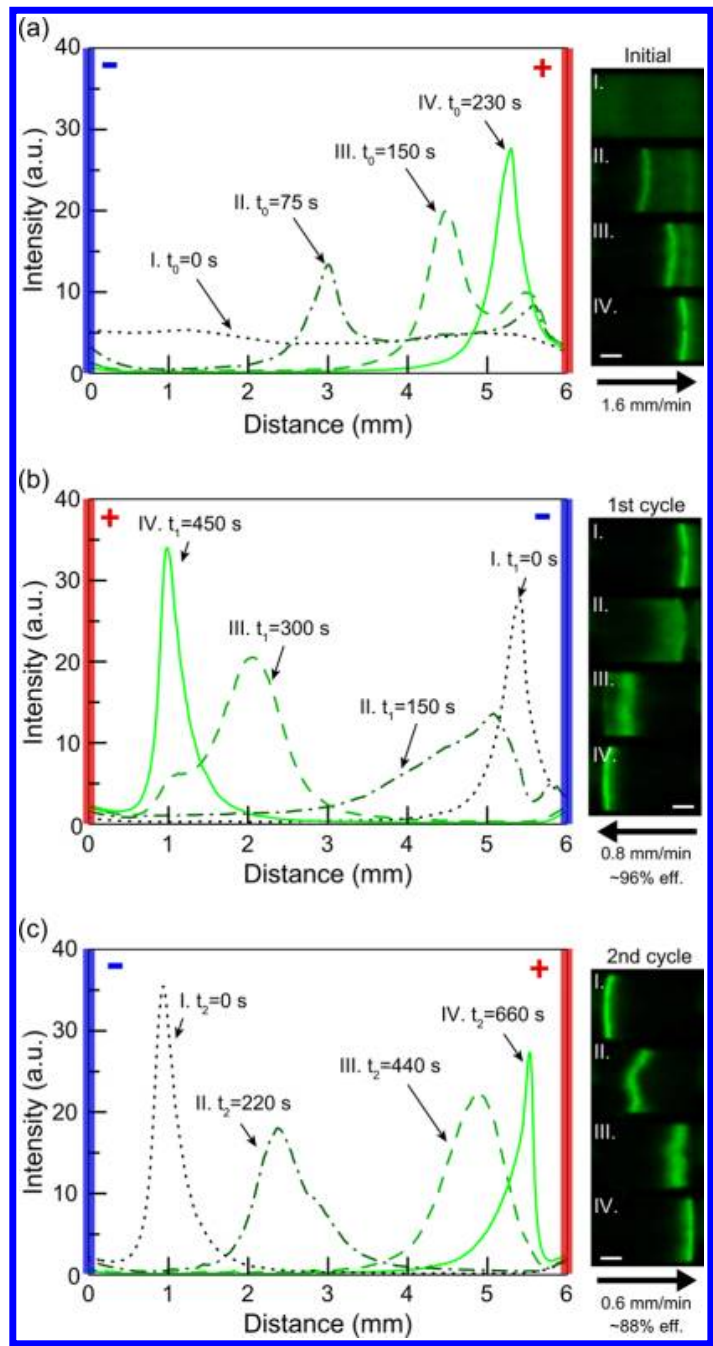

Figure 4. Active transport using the external device. (a) Initial concentration of fluorescent tracer from the cathode (negatively polarized membrane denoted by the blue band, "-") to the anode (positively polarized membrane denoted by the red band, "+") at a rate of $1.6 \mathrm{~mm} / \mathrm{min}$, under an applied voltage of $50 \mathrm{~V}$. (b) The concentrated band is transported to the opposite side upon reversal of membrane polarities, at a rate of $0.8 \mathrm{~mm} / \mathrm{min}$ and $\sim 96 \%$ efficiency. (c) Membrane polarities are reversed again, and the concentrated band is transported to the opposite side, at a rate of $0.6 \mathrm{~mm} / \mathrm{min}$ and $\sim 88 \%$ efficiency. Contrast enhanced images are shown for the transport process at indicated time points. Scale bars are $2 \mathrm{~mm}$.

centimeter distances with minimal loss, enabled by external Nafion nanoporous membranes.

Active Concentration and Transport Using the InPaper Device. Experimental results for fluorescent tracer concentration using the in-paper concentration device are shown in Figure 5. Analogous to the circular geometry of the external concentration device (Figure 1), the in-paper device has an outer semicircular membrane enclosing the sample region (with a diameter of $8 \mathrm{~mm}$ ). For an applied voltage of 50 $\mathrm{V}$, a $15 \mu \mathrm{L}$ sample of fluorescein solution is concentrated after 8.5 min (movie 3 in the Supporting Information). In Figure 5a, the area of the concentrated spot is $1.3 \mathrm{~mm}^{2}$ as compared to an initial area of $32 \mathrm{~mm}^{2}$, giving a surface area ratio of 25 -fold. The measured maximum peak concentration factor based on fluorescence intensity is 22 -fold (i.e., $\sim 88 \%$ of the upper limit), as shown in Figure 5b. Thus, concentration efficiencies
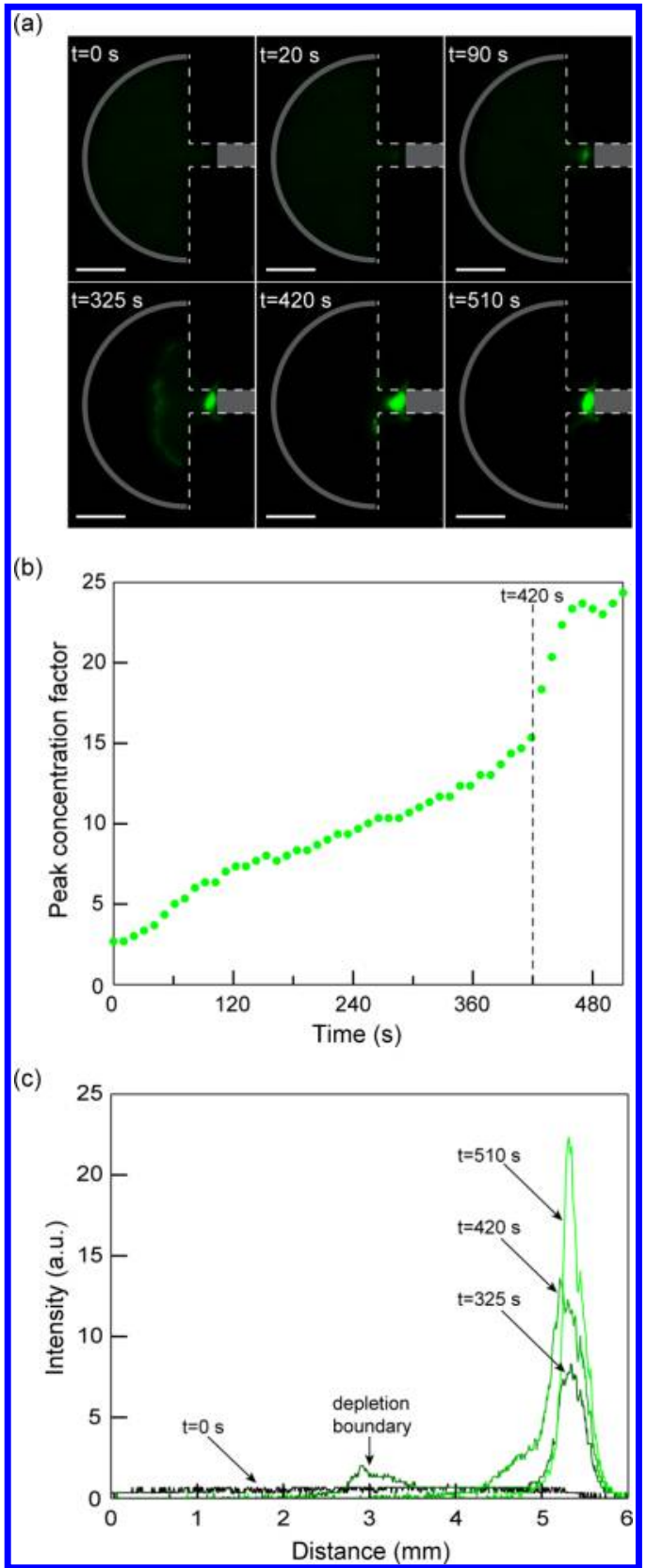

Figure 5. Active concentration using the in-paper device. (a) Contrast enhanced images of fluorescent tracer concentration under an applied voltage of $50 \mathrm{~V}$. Patterned inner and outer nanoporous membranes are indicated by the rectangle and arc, respectively. Patterned wax boundaries are indicated by the dashed lines. (b) Time evolution of peak concentration factor. The maximum value reaches 22 -fold at $t=$ $510 \mathrm{~s}$. Merging of the depletion and enrichment regions is indicated by the dashed line at $t=420 \mathrm{~s}$. (c) Intensity profiles at selected time points, as indicated. Scale bars are $2 \mathrm{~mm}$.

of both the in-paper and the external approaches are high ( $\sim 88 \%$ here as compared to $\sim 91 \%$ for the external device). Together, the results in Figure 5 demonstrate the concentration of analytes using the in-paper approach, with substantial improvement in signal intensity. Notably, nanoporous membrane material patterned into paper can both define flow regions and enable ICP concentration.

A noteworthy alternative to the No. 1 paper employed here is nitrocellulose membrane, which is commonly used in commercial assays. For completeness, we patterned nano- 
porous membranes into nitrocellulose membrane to concentrate fluorescent tracer, resulting in a 16 -fold concentration comparable to the 22 -fold concentration achieved in No. 1 paper (full details are provided in Figure S2 of the Supporting Information).

Experimental results for fluorescent tracer transport using the in-paper transport device are shown in Figure 6. For an applied

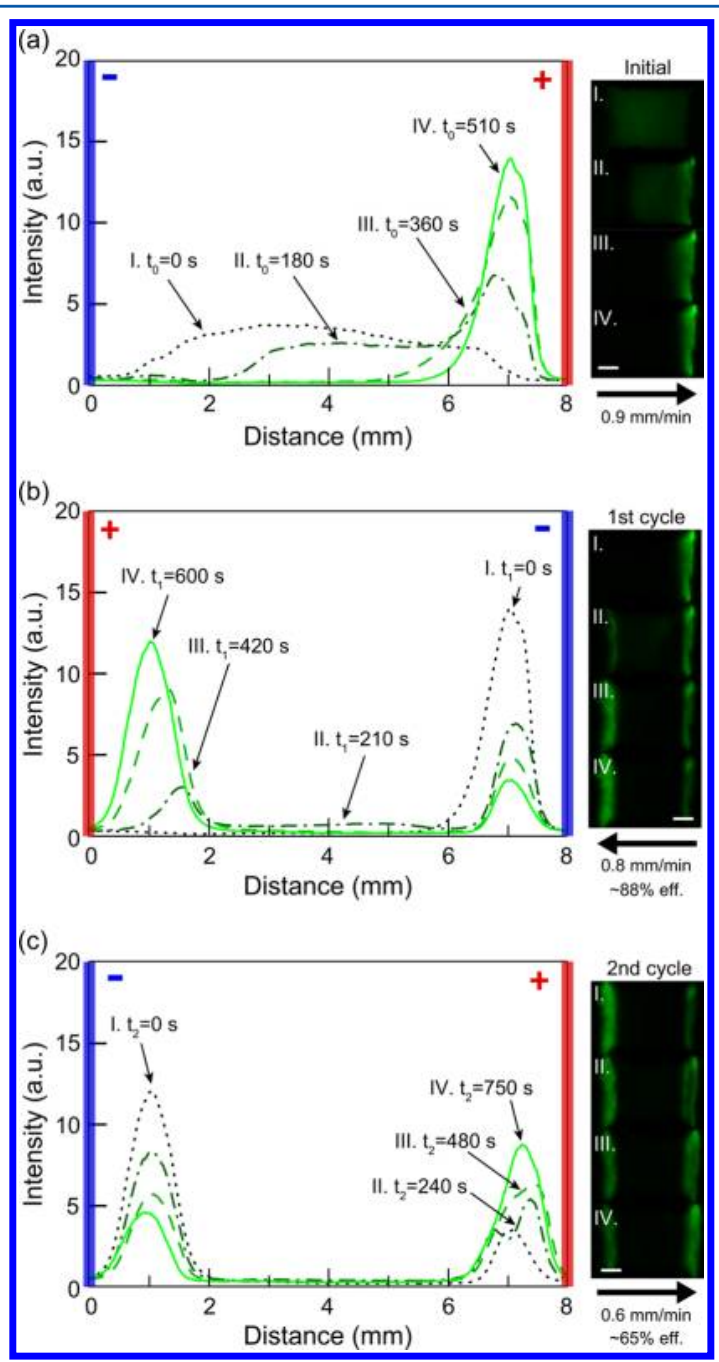

Figure 6. Active transport using the in-paper device. (a) Initial concentration of fluorescent tracer from the cathode (blue band, "-") to the anode (red band, "+") at a rate of $0.9 \mathrm{~mm} / \mathrm{min}$, under an applied voltage of $50 \mathrm{~V}$. (b) The concentrated band is transported to the opposite side upon switching membrane polarities, at a rate of 0.8 $\mathrm{mm} / \mathrm{min}$ and $\sim 88 \%$ efficiency. (c) Membrane polarities are reversed again, and the concentrated band is transported to the opposite side, at a rate of $0.6 \mathrm{~mm} / \mathrm{min}$ and $\sim 65 \%$ efficiency. Contrast enhanced images are shown for the transport process at indicated time points. Scale bars are $2 \mathrm{~mm}$.

voltage of $50 \mathrm{~V}$, a $5 \mu \mathrm{L}$ sample of fluorescein solution is transported multiple cycles in an $8 \mathrm{~mm} \times 3 \mathrm{~mm}$ paper channel (movie 4 in the Supporting Information). The sample is initially concentrated at an average rate of $0.9 \mathrm{~mm} / \mathrm{min}$, as shown in Figure 6a. Following this step, the concentrated sample is transported multiple cycles by switching the polarity of the membranes, as shown in Figure $6 \mathrm{~b}$ and c. This back and forth transport is achieved over the $\sim 8 \mathrm{~mm}$ distance (total of $\sim 24 \mathrm{~mm}$ ) with relatively little loss, albeit more than in the external device case. Specifically, the fwhm values for the initial and two subsequent transport cases are $0.79,0.84$, and 0.82 $\mathrm{mm}$.

Transport efficiencies of $\sim 88 \%$ and $\sim 65 \%$ are observed for the first and second cycles, respectively (with transport efficiency as defined earlier). A key cause of the reduction in transport efficiency is fluorescent tracer left behind during each transport cycle, as indicated by the remaining peaks at the cathodic side in Figure $6 \mathrm{~b}$ and $6 \mathrm{c}$. We attribute these remaining peaks to evaporation, and, specifically, sample stranded as a result of evaporation. To verify the role of evaporation here, a separate test was conducted with a glass coverslip to minimize fluid loss in the sample region. The results of the test yielded consistent transport efficiencies of $\sim 90 \%$ and average transport rates of $1.1 \mathrm{~mm} / \mathrm{min}$ for both the first and the second cycles (Figure S3 in the Supporting Information). Thus, the in-paper device can be run without a coverslip as demonstrated in Figure 6; however, if longer run times and/or higher transport efficiencies are required, a coverslip evaporation barrier is recommended. Collectively, the results in Figure 6 demonstrate the directional transport of analytes in paper, over centimeter distances, using patterned nanoporous membranes.

Protein and Colored Dye Concentration Using the InPaper Concentration Device. To demonstrate the diagnostic applicability of the devices developed here, the in-paper concentration device is used to concentrate protein (i.e., FITCalbumin) and dye (i.e., bromocresol green dye). The geometry of the device is slightly modified such that wax boundaries are angled to provide better focusing in the sample region, as shown in Figure 7b. FITC-albumin is used as a model protein, enabling quantitative imaging at low concentrations, and the dye is a typical model of analytes in paper-based colorimetric assays as visualized with a desktop scanner.

The limit of detection (LOD) curves for FITC-albumin and bromocresol green dye before and after concentration are shown in Figure 7. The intensity value at each molar concentration is averaged over $n=3$ independent measurements with error bars representing one standard deviation. The LOD for each analyte is given as the molar concentration corresponding to the intensity value that is three standard deviations from the control intensity value. In Figure $7 \mathrm{a}$, the LOD for FITC-albumin before concentration is $\sim 10$ and $\sim 2$ $\mathrm{pmol} / \mathrm{mL}$ after concentration. Similarly in Figure $7 \mathrm{~b}$, the LOD for bromocresol green dye before concentration is $\sim 40$ and $\sim 10 \mu \mathrm{M}$ after concentration. These results demonstrate an extension of the limit of detection with implications for the growing field of paper-based (colorimetric) assays.

\section{CONCLUSIONS}

We demonstrated active concentration and transport of analytes in fully wet paper-based assays by leveraging nanoporous material and ion concentration polarization. This work is distinct from the rapidly growing literature on paperbased microfluidics in that it offers unprecedented analyte control, independent of wetting history and capillary forces. In the in-paper devices, this functionality was enabled by patterned nanoporous material that (1) acts as a hydrophobic barrier, much like wax, to define fluidic reservoirs and channels, and (2) creates the micronano interface necessary for ICP phenomena. Experimental results yielded up to 40 -fold concentration and up to $96 \%$ transport efficiency of a fluorescent tracer. Directional transport of the tracer was achieved over centimeters with consistent peak shape and minimal loss. Most notably, the 


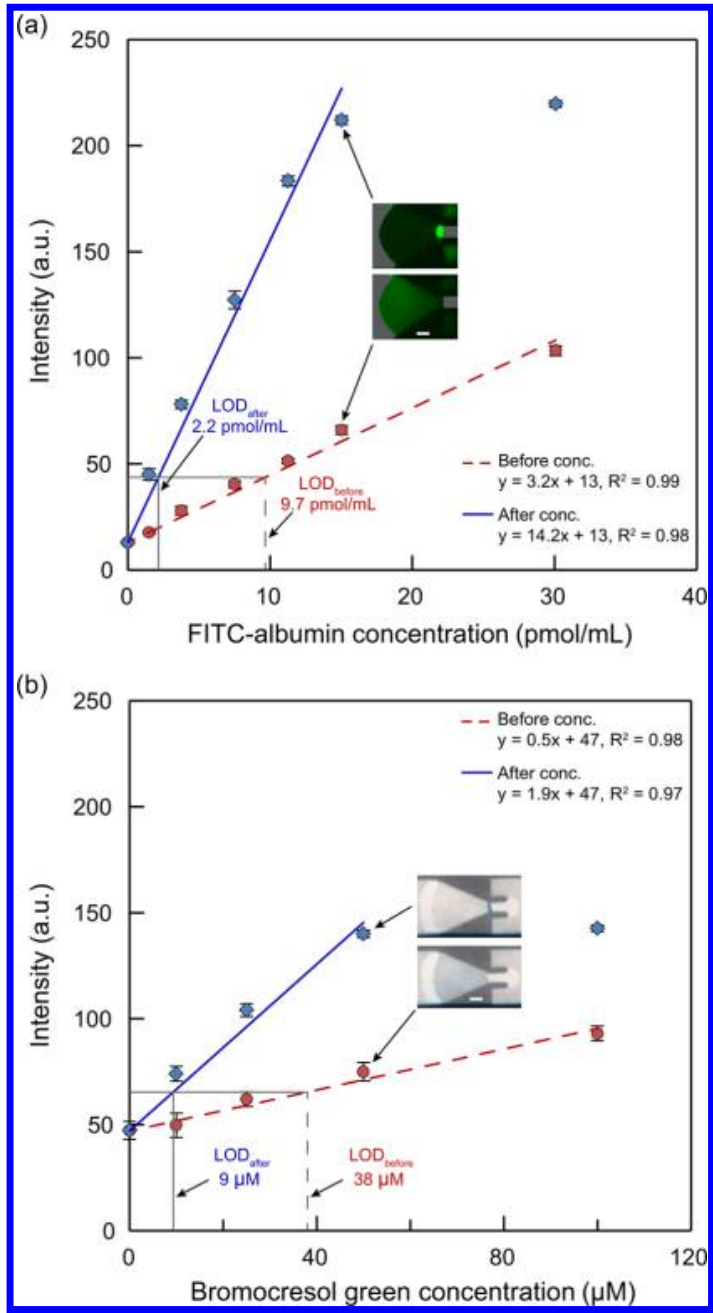

Figure 7. Concentration of a model protein and colored dye using the in-paper concentration device. (a) Limit of detection (LOD) curves for FITC-albumin before (dashed) and after (solid) concentration. The LOD before concentration is $\sim 10$ and $\sim 2 \mathrm{pmol} / \mathrm{mL}$ after concentration. Device images are shown inset, with patterned membranes indicated by the arc and rectangle. (b) LOD curves for bromocresol dye before (dashed) and after (solid) concentration. The LOD before concentration is $\sim 40 \mu \mathrm{M}$ and $\sim 10 \mu \mathrm{M}$ after concentration. Device images are shown inset. Each intensity value is averaged over $n=3$ independent measurements with error bars representing one standard deviation. Scale bars are $2 \mathrm{~mm}$.

limits of detection for protein and colored dye were improved using the in-paper approach. Collectively, these results demonstrate a major advance in analyte concentration and manipulation for the growing field of low-cost paper-based assays.

\section{ASSOCIATED CONTENT}

\section{S Supporting Information}

Movies 1-4: Fluorescent tracer concentration and transport using the external and in-paper devices. Figure S1: Details for the fabrication and assembly of the external devices. Figure S2: In-paper concentration of fluorescent tracer in nitrocellulose membrane. Figure S3: Fluorescent tracer transport using the inpaper device with a coverslip over the sample region. Tables S1 and S2: Material cost and fabrication time requirements for the external and in-paper devices, respectively. This material is available free of charge via the Internet at http://pubs.acs.org.

\section{AUTHOR INFORMATION}

\section{Corresponding Authors}

*E-mail: brendan.macdonald@uoit.ca.

*E-mail: sinton@mie.utoronto.ca.

\section{Author Contributions}

M.M.G., B.D.M., and D.S. designed research; M.M.G. and P.Z. performed research; and M.M.G., P.Z., and B.D.M. analyzed data. The manuscript was written through contributions of all authors. All authors have given approval to the final version of the manuscript.

\section{Notes}

The authors declare no competing financial interest.

\section{ACKNOWLEDGMENTS}

We gratefully acknowledge a Natural Sciences and Engineering Research Council of Canada Postgraduate Scholarship (NSERC-PGSD) to M.M.G. The work was also enabled by funding from Grand Challenges Canada through the Point of Care Diagnostics Program, infrastructure funding from Canada Foundation for Innovation and the Ontario Research Fund (CFI and ORF), and ongoing support from NSERC.

\section{REFERENCES}

(1) Hu, L.; Wu, H.; La Mantia, F.; Yang, Y.; Cui, Y. ACS Nano 2010, $4,5843-5848$.

(2) Thom, N. K.; Yeung, K.; Pillion, M. B.; Phillips, S. T. Lab Chip 2012, 12, 1768-1770.

(3) Gui, Z.; Zhu, H.; Gillette, E.; Han, X.; Rubloff, G. W.; Hu, L.; Lee, S. B. ACS Nano 2013, 7, 6037-6046.

(4) Kurra, N.; Kulkarni, G. U. Lab Chip 2013, 13, 2866-2873.

(5) Tobjörk, D.; Österbacka, R. Adv. Mater. 2011, 23, 1935-1961.

(6) Zhao, W.; Ali, M. M.; Aguirre, S. D.; Brook, M. A.; Li, Y. Anal. Chem. 2008, 80, 8431-8437.

(7) Ngo, Y. H.; Li, D.; Simon, G. P.; Garnier, G. Adv. Colloid Interface Sci. 2011, 163, 23-38.

(8) Veigas, B.; Jacob, J. M.; Costa, M. N.; Santos, D. S.; Viveiros, M.; Inacio, J.; Martins, R.; Barquinha, P.; Fortunato, E.; Baptista, P. V. Lab Chip 2012, 12, 4802-4808.

(9) Liu, Q.; Wang, J.; Wang, B.; Li, Z.; Huang, H.; Li, C.; Yu, X.; Chu, P. K. Biosens. Bioelectron. 2014, 54, 128-134.

(10) Martinez, A. W.; Phillips, S. T.; Whitesides, G. M.; Carrilho, E. Anal. Chem. 2010, 82, 3-10.

(11) Li, X.; Ballerini, D. R.; Shen, W. Biomicrofluidics 2012, 6.

(12) Yetisen, A. K.; Akram, M. S.; Lowe, C. R. Lab Chip 2013, 13, 2210-2251.

(13) Gong, M. M.; MacDonald, B. D.; Nguyen, T. V.; Van Nguyen, K.; Sinton, D. Lab Chip 2014, 14, 957-963.

(14) Nery, E. W.; Kubota, L. T. Anal. Bioanal. Chem. 2013, 405, $7573-7595$

(15) Böhm, A.; Carstens, F.; Trieb, C.; Schabel, S.; Biesalski, M. Microfluid. Nanofluid. 2014, 1-11.

(16) Fu, E.; Kauffman, P.; Lutz, B.; Yager, P. Sens. Actuators, B: Chem. 2010, 149, 325-328.

(17) Fu, E.; Liang, T.; Houghtaling, J.; Ramachandran, S.; Ramsey, S. A.; Lutz, B.; Yager, P. Anal. Chem. 2011, 83, 7941-7946.

(18) Hu, J.; Wang, L.; Li, F.; Han, Y. L.; Lin, M.; Lu, T. J.; Xu, F. Lab Chip 2013, 13, 4352-4357.

(19) Allen, P. B.; Arshad, S. A.; Li, B.; Chen, X.; Ellington, A. D. Lab Chip 2012, 12, 2951-2958.

(20) Yu, W. W.; White, I. M. Analust 2013, 138, 1020-1025.

(21) Fu, E.; Lutz, B.; Kauffman, P.; Yager, P. Lab Chip 2010, 10, 918-920.

(22) Fu, E.; Ramsey, S.; Kauffman, P.; Lutz, B.; Yager, P. Microfluid. Nanofluid. 2011, 10, 29-35.

(23) Li, X.; Tian, J.; Nguyen, T.; Shen, W. Anal. Chem. 2008, 80, 9131-9134. 
(24) Li, X.; Zwanenburg, P.; Liu, X. Lab Chip 2013, 13, 2609-2614.

(25) Liu, X. Y.; Cheng, C. M.; Martinez, A. W.; Mirica, K. A.; Li, X. J.; Phillips, S. T.; Mascareñas, M.; Whitesides, G. M. A portable microfluidic paper-based device for ELISA. Micro Electro Mechanical Systems (MEMS), 2011 IEEE 24th International Conference; 2011; pp $75-78$.

(26) Lutz, B.; Liang, T.; Fu, E.; Ramachandran, S.; Kauffman, P.; Yager, P. Lab Chip 2013, 13, 2840-2847.

(27) Toley, B. J.; McKenzie, B.; Liang, T.; Buser, J. R.; Yager, P.; Fu, E. Anal. Chem. 2013, 85, 11545-11552.

(28) Noh, H.; Phillips, S. T. Anal. Chem. 2010, 82, 4181-4187.

(29) Jahanshahi-Anbuhi, S.; Henry, A.; Leung, V.; Sicard, C.; Pennings, K.; Pelton, R.; Brennan, J. D.; Filipe, C. D. M. Lab Chip 2014, 14, 229-236.

(30) Houghtaling, J.; Liang, T.; Thiessen, G.; Fu, E. Anal. Chem. 2013, 85, 11201-11204.

(31) Kunkel, H. G.; Tiselius, A. I. Gen. Phvsiol. 1951, 35, 89-118.

(32) Fobel, R.; Kirby, A. E.; Ng, A. H. C.; Farnood, R. R.; Wheeler, A. R. Adv. Mater. 2014.

(33) Höltzel, A.; Tallarek, U. J. Sep. Sci. 2007, 30, 1398-1419.

(34) Pu, Q. S.; Yun, J. S.; Temkin, H.; Liu, S. R. Nano Lett. 2004, 4, 1099-1103.

(35) Wang, Y.; Stevens, A. L.; Han, J. Anal. Chem. 2005, 77, 42934299.

(36) Lee, J. H.; Song, Y.; Han, J. Lab Chip 2008, 8, 596-601.

(37) Lee, J. H.; Song, Y.; Tannenbaum, S. R.; Han, J. Anal. Chem. 2008, 80, 3198-3204.

(38) Scarff, B.; Escobedo, C.; Sinton, D. Lab Chip 2011, 11, 11021109.

(39) Ko, S. H.; Song, Y.; Kim, S. J.; Kim, M.; Han, J.; Kang, K. H. Lab Chip 2012, 12, 4472-4482.

(40) Pevarnik, M.; Schiel, M.; Yoshimatsu, K.; Vlassiouk, I. V.; Kwon, J. S.; Shea, K. J.; Siwy, Z. S. ACS Nano 2013, 7, 3720-3728.

(41) Jeon, H.; Lee, H.; Kang, K. H.; Lim, G. Sci. Rep. 2013, 3.

(42) Kim, S. J.; Ko, S. H.; Kang, K. H.; Han, J. Nat. Nanotechnol. 2010, 5, 297-301.

(43) MacDonald, B. D.; Gong, M. M.; Zhang, P.; Sinton, D. Lab Chip 2014, 14, 681-685.

(44) Mai, J.; Miller, H.; Hatch, A. V. ACS Nano 2012, 6, 1020610215 .

(45) Mauritz, K. A.; Moore, R. B. Chem. Rev. 2004, 104, 4535-4585.

(46) Emmart, E. W. Arch. Biochem. Biophvs. 1958, 73, 1-8. 